\title{
The Behaviour of Colour Terms in Jordanian Arabic: Lexical and Grammatical Evidence
}

\author{
Mohammad Issa Mehawesh ${ }^{1}$ \\ Abdullah Ahmad Jaradat ${ }^{2}$ \\ Alaeddin Sadeq ${ }^{3}$ \\ 1 Department of English Language and Translation, Zarqa University, Zarqa, Jordan \\ 2 Dept. of English language and Literature, Hashemite University, Zarqa, Jordan \\ 3. Department of English Language, Zarqa University, Post address: 132222, Zarqa 13132- Jordan \\ Correspondence: Mohammad Issa Mehawesh, Faculty of Arts, Department of English Language and Translation \\ Zarqa University, PO box 132222, Zarqa 13132, Jordan. Tel: 962-7-9749-2506. E-mail: mohammedissa33@yahoo.com
}

\section{Doi:10.5901/mjss.2015.v6n6s4p44}

\section{Abstract}

The purpose of this paper is to provide grammatical and lexical evidence in support of primary color terms in Jordanian Arabic, henceforth, JA. The general framework is to investigate the behavior of basic color terms in some lexical or grammatical processes and to prove that their behavior supports the division proposed by Kay and McDanial (1978) between primary and derived color terms. The evidence is distilled from various sources including: the templates used for the two groups of color terms, the feminine templates utilized in each group; the plural templates used in each group; reduplication; the parts of speech derived from each color; the variety of color terms; and the frequency of each color in daily usage.

Keywords: Behavior, Colour, Grammatical, Lexical.

\section{Introduction}

Despite the expansive literature on basic colour terms in English including Crawford (1982) and Moss (1989) among others, the behaviour of basic colour terms in Arabic has been scarcely investigated. Maffi (1990) focused on the number of parts of speech derived from a basic colour. Maffi has found that some colours show more derivatives than others. Moro (2007) studied colour terms in English-lexifier Atlantic creoles. She utilized dictionary entries per colour. She has found that with the exception of grey, dictionary entries reflect the basic colour patterns suggested by Berlin and Kay. Bender (1983) discussed the colour terms that can be encoded special meanings with special reference to skin. Omar (1997) wrote a book about the relationship between language and colour in which he summarized all the theoretical issues related to colours including the Berlin and Kay's theory of basic colour terms. Al-Adaileh (2012) discussed the connotations of Arabic colour terms in Jordanian Arabic. Rabab'ah and Al-Saidat (2014) discussed the conceptual and the connotative meanings of the colours black and white in Jordanian Arabic. They have that participants interpret the target expressions according to their connotations not literal meanings. They have also found that the colour white has generally speaking good connotations, while the colour black has bad or negative connotations. Hence there has been a paucity of research on basic colour terms in Arabic, and this paper closes the gap in the literature review.

This study investigates the behaviour of basic colour terms in Jordanian Arabic. It proves that the division proposed by Berlin and Kay (1968) between primary colour terms and derived colour terms applies to Jordanian Arabic. Genuine Evidence for this claim will be given from different sources including: the colour term templates, gender markers, the plural forms, the parts of speech derived from the colour terms, reduplication, the variety of colour terms, the frequency of colour terms in a daily use and the metaphorical or the connotative meaning each colour carries.

\section{Theoretical Background}

This study relies upon a major article by Kay and McDenial (1978) in which they presented an important revision to Berlin and Kay's (1969) original theory of basic colour terms and colour universals. Moreover, the findings of the study supports the distinction presented between primary colour terms which include white, black, red, yellow, green and blue and derived colour terms which include brown, pink, purple, orange and gray. 
Relying upon their survey of 20 unrelated languages, Berlin and Kay (1969) proposed that there are 11 basic colour terms which can be considered as universal colours. Interestingly, these colours appeared in a predictable order. Berlin and Kay presented the colours in the implicational hierarchy given in Figure 1 below:

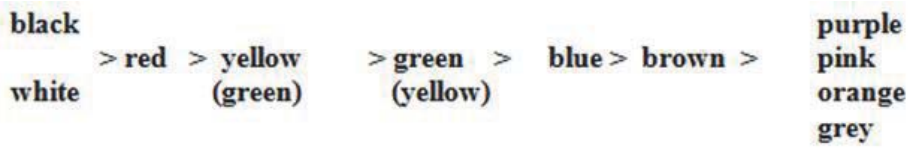

Figure 1: The hierarchy of basic colour terms

In this hierarchy, the presence of any colour term to the right implicates the presence of the colour term(s) to the left. For example, the presence of the colour term "blue" in a language implies the presence of the colour terms "white", "black", "red", "green", and "yellow" in that same language. Furthermore, they claim that this hierarchy is a diachronic one in the sense that it describes the evolution of basic colour terms. (Davies, Roling, Corbett, Xoagub, and Xoagub, 1998) say, 'Languages differ because they are at different stages of the evolution of colour terms' ( $p$ 182). The picture is the following: any language, through the process of developing its colour inventory, encodes colour terms for black and white first. Then, at the second stage the language encodes a term for red. At a later stage, the language encodes a term for green or yellow. If green is encoded first then yellow will be the fifth colour to encode and vice versa. To sum up, the present hierarchy is synchronic in the sense that it describes the present colour inventory in a language, and diachronic since it accounts for the evolutional stages of encoding colour terms in the world's languages.

Berlin and Kay (1969) came up with a set of criteria or characteristics for basic colour terms. The criteria are given below:

(2) The criteria of basic colour terms:

a. Basic colour terms should be morphologically simple i.e. made of one lexeme (thus yellowish-green is not basic since its meaning is predictable from the meaning of its parts).

b. The signification of basic colour terms is not included in any other colour (unlike crimson which is a kind of red)

c. The use of basic colour terms is not limited to a narrow range of objects (thus Blonde is not a basic colour since it is restricted to hair and complexion)

d. They are psychologically salient in everyday use.

With regard the salience of a colour, Berlin and Kay $(1969, p$ 6) said that a colour can be considered salient "if (a) it tends to occur at the top of elicited lists of colour terms; (b) it has a stable reference across speakers and across occasions of use; and (c) it occurs in the ideolects of all informants.

Furthermore, Berlin and Kay (1969) introduced a number of subsidiary criteria to account for some doubtful cases. On the basis of these criteria, some colours terms can be suspects for being basic colour terms and on the basis of which some colour terms can be eliminated. These criteria can be termed 'exclusive criteria' in the sense that any colour term that does not meet these criteria will be excluded from being a basic colour.

(3) Exclusive criteria:

a. Distributional potential: i.e. any doubtful colour should have the same frequency of the already-established colour terms.

b. A colour which is also the name of an object having that colour.

c. Loan words are more likely to be excluded from being basic colour terms.

d. Morphological complexity is utilized to exclude some colours that pass the mono-lexemic criterion.

Arabic, represented by Lebanese Arabic, was one of the languages included in Berlin and Kay's survey. It was considered a stage-VII language which contains the 11 basic colour terms suggested by the researchers. Jordanian Arabic is no different from Lebanese Arabic as it contains the full list of the 11 basic colours terms. A list of the 11 basic colour terms in Jordanian Arabic is given below:

(4) Basic colour terms in Jordanian Arabic:

?IbjaD 'white'
?Iswad 'black'
?Iћmar 'red'
?IXDar 'green'


PISfar 'yellow'
PIzrag 'blue'
bunni 'brown'
burudgaani 'orange'
zahri 'pink'
ramaadi 'gray'
banafsadzI 'purple'
In table (1) below, the colours are judged against the main set of criteria introduced by Berlin and Kay:

Table (1): The basic colour terms in Jordanian Arabic judged against the criteria:

\begin{tabular}{||l||c|c|c|c||}
\hline \hline & $\begin{array}{c}\text { Morphologically } \\
\text { simple }\end{array}$ & $\begin{array}{c}\text { Signification not included in } \\
\text { other colours }\end{array}$ & $\begin{array}{c}\text { Use not limited to a narrow } \\
\text { range of objects }\end{array}$ & $\begin{array}{c}\text { Salient in } \\
\text { everyday use }\end{array}$ \\
\hline \hline IbjaD & + & + & + & + \\
\hline ISWad & + & + & + & + \\
\hline Ihmar & + & + & + & + \\
\hline IXDar & + & + & + & + \\
\hline ISfar & + & + & + & + \\
\hline IZrag & + & + & + & + \\
\hline binnI & - & + & + & + \\
\hline burudgaanI & - & + & + & + \\
\hline ZahrI & - & + & + & + \\
\hline ramaadI & - & + & + & + \\
\hline banafsad3I & - & + & \multicolumn{2}{|l}{} \\
\hline
\end{tabular}

The table shows clearly that the last five colours do not pass the first criterion though they clearly pass all the other criteria.

Major developments to Berlin and Kay's original theory come from Kay and McDaniel (1978) and Kay, Berlin and Merrifield (1991), though the only relevant development for the purpose of the study comes from the first article. Relying on semantic universals and the neuro-physical bases of colour perception, they adopted the fuzzy set theory which nullifies the discrete features of colours i.e. there are no clear-cut boundaries between colours. The fuzzy set theory recognizes degrees of membership in the category of colours. However, the major proposal that concerns us here is the division between primary colour terms which include black, white, red, yellow, green, and blue and derived colour terms which include brown, pink, purple, orange, and gray. The first group of colours was named primary since, according to Kay and McDaniel (1978), the semantic category is identical to the neural response categories. The second group was named derived in the sense that they result from an overlap or an intersection between two basic colours: brown is found in the region where yellow and black overlap; gray where white and black overlap; pink where red and white overlap; orange where red and yellow overlap and purple where red and blue overlap. Colours that are not included in these two groups were called secondary colours. These colours do not exhibit the main characteristics.

\section{Discussion}

Primary colours i.e. black, white, red, green, yellow, and blue in JA seem to exhibit all the main characteristics while derived colours satisfy most of the criteria presented as they fail the morphology requirement as they have an additional morpheme which is the derivational suffix indicating relation. This last feature makes them morphologically complex. Colours that are not included in these two groups are called secondary colours. Table (1) above clearly manifests this division.

I claim that the behaviour of colour terms in JA supports the division proposed between primary colour terms and derived colour terms. Primary colour terms behave differently from derived ones. Evidence for this claim will be given from different sources including: the colour term templates, gender markers, the plural forms, the parts of speech derived from the colour terms, reduplication, the variety of colour terms, the frequency of colour terms in a daily use and the 
metaphorical or the connotative meaning each colour carries.

\subsection{Colour Terms Templates}

The first thing to notice is the different templates of the two groups of colour terms. Primary colour terms follow a stable template made of two closed syllables CVC-CVC as given in the list of colour terms in JA in (4) above. Omar (1997) nullifies the restriction of this template to basic colours on the basis that other colours which are not basic have the same template such as Pasmar 'brown complexion' and Parmad 'gray'. However, these colours fail at least two other criteria: their meaning is included in another colour's meaning as in the case of Parmad 'gray' whose meaning is included in ramadi 'gray', and they are restricted in use as in the case of Pasmar 'brown complexion' which can only be used to refer to complexion. Moreover, these examples are for Standard Arabic, which is not understudy in this paper. However, when these 11 basic colour terms are considered, only primary colours have this template. Derived colour terms, on the other hand, have different shapes depending on the source noun they are derived from. The only feature shared between the derived colours is the "-I" ending which is a derivational suffix as shown in (4) above.

Moreover, derived colours are literally derived. They are made of two parts: a collective noun taken from real life and '-I' which is a derivational suffix meaning 'related to'; a fact which explains why they failed the first criterion. The derived colours can be analyzed as follows:

(5) A morphological analysis of derived colour terms

a. binni "brown" bunn 'coffee' + '-I' "related to"

b. burudgaan I "orange" burudgaan 'orange' + '-I' "related to"

c. zahri "pink" zahr 'flowers' + '-I' "related to"

d. ramaadr "gray" ramaad 'ash' + '-I' "related to'

e. banafsadzI "purple" banafsadz 'violet' + '-I' "related to"

As the analysis above shows, derived colours are made of two lexemes; a fact which contradicts with one of Berlin and Kay's criteria. Moreover, they are derived from objects which do not express a colour or even used to express a colour. Nevertheless, these colours comply with the other three criteria in the sense that they are salient in everyday life; that their signification is not included in any other colour; and that they are not limited in use. On a scale, we cannot nullify the basicness of these colours on the basis of the absence of one criterion.

In this context, it is better to reanalyze the derived colours into their components. All derived colours as well as secondary colours are derived from collective nouns by virtue of the addition of the relational suffix ' $\mathrm{I}$ ' whose main function in Arabic is to derive identity, nationality or relation to a source. Interestingly enough, these colour terms are derived using collective nouns, some of which have been dropped from use in real life and some have been replaced with other expressions. Table (2) below presents the collective name from which the colours are derived, the most common expression used in real life, as well as the singular and the plural forms:

Table (2): Resources of the derived nouns:

\begin{tabular}{||l|l|l|l|l||}
\hline Colour & The collective noun & The most common expression & Singular & Plural \\
\hline \hline binnI & $\begin{array}{l}\text { bunn } \\
\text { 'coffee/ the product' }\end{array}$ & $\begin{array}{l}\text { 'qahwa' } \\
\text { 'ground coffee' }\end{array}$ & $\begin{array}{l}\text { habit qahwa } \\
\text { 'a coffee bean' }\end{array}$ & $\begin{array}{l}\text { habaat } \\
\text { 'beans' }\end{array}$ \\
\hline Zahri & $\begin{array}{l}\text { Zahur } \\
\text { 'flowers' }\end{array}$ & $\begin{array}{l}\text { Zahra 'a flower' } \\
\text { wardeh 'a rose' }\end{array}$ & $\begin{array}{l}\text { zahraat 'flowers' } \\
\text { wardaat 'roses' }\end{array}$ \\
\hline ramaadI & $\begin{array}{l}\text { Ramaad } \\
\text { 'ash' }\end{array}$ & $\begin{array}{l}\text { ramaad } \\
\text { 'ash' }\end{array}$ & $\begin{array}{l}\text { ramaadeh } \\
\text { 'an ash' }\end{array}$ & ramaadaat 'ashes' \\
\hline banafsadzI & $\begin{array}{l}\text { Banafsad3 } \\
\text { 'violet' }\end{array}$ & $\begin{array}{l}\text { banafsad3 } \\
\text { 'violet' }\end{array}$ & $\begin{array}{l}\text { 'a violet' } \\
\text { 'arudganeh }\end{array}$ & $\begin{array}{l}\text { banafsadzaat } \\
\text { 'violets' }\end{array}$ \\
\hline burudgaan, & burudgaan \\
'orange' & burudgaan 'orange' & $\begin{array}{l}\text { burudganaat } \\
\text { 'oranges' }\end{array}$ \\
\hline
\end{tabular}

The following remarks are deduced from the data given above. First: the word bunn is not used in real life except in the colour term and in media when they talk about the prices of coffee i.e. the product. The word zahur is not used in real life 
except in the colour term; it is usually replaced with ward 'roses'. Finally, the colour ramaad $I$ is most likely replaced with the colour PrSaaSI 'related to lead' or sat SanI 'related to ash'.

\subsection{Feminine Marking}

Both primary and derived colour terms can be marked for gender. Still, the two categories show different markers for gender. Primary colour terms can be turned feminine by adding the suffix "-a" to the root, while derived colour terms can be turned feminine be using the regular feminine marker "-eh" to the root. The masculine as well as the feminine forms are given below in (6):

(6) Feminine marking

a. Primary colour terms: Male Female

$$
\begin{aligned}
& \text { PibjaD beiD-a } \\
& \text { ?iswad soud-a } \\
& \text { ?ihmar hamr-a } \\
& \text { ?ISfar Safr-a } \\
& \text { ?ixDar xaDr-a } \\
& \text { ?izrag zarg-a }
\end{aligned}
$$

b. Derived colour terms

$$
\begin{aligned}
& \text { binni binnijj-eh } \\
& \text { zahri zahrijj-eh } \\
& \text { ramaadi ramaadijj-eh } \\
& \text { banafsadzI banafsadzijj-eh } \\
& \text { burudgaani burudgaanij-eh }
\end{aligned}
$$

The difference in feminine marker is due to the fact that the marker used with primary colour terms is colourspecific i.e. this gender marker is only used with these colour terms and accidentally with bodily defects as in ?rirad3 limp 'masculine' Sardza limp 'feminine'. Derived colour terms, on the other hand, exhibit the regular feminine marker. Consider, the colour terms for red and brown when used with the word 'car' which is considered feminine in JA:

(7) Two colour terms with the word 'car' in JA:
a. a. sajjaarah hamr-a car red 'a red car'
b. b. sajjaarah binnijj-eh car brown 'a brown car'

This difference in feminine marker can be given as an additional proof on the division between primary and derived colour terms.

\subsection{Pluralization}

The two groups of colour terms exhibit variation with regard to pluralization. Primary colour terms show a fixed form that can be utilized for both genders and for both humans and nonhumans. The plural forms are given in (8) below alongside the singular masculine and feminine forms:

(8) The plural forms of primary colours terms:

$\begin{array}{lll}\frac{\text { masculine }}{\text { PibjaD }} & \frac{\text { feminine }}{\text { beiDa }} & \text { plural } \\ \text { biiD } \\ \text { PIswad } & \text { souda } & \text { suud } \\ \text { PIћmar } & \text { hamra } & \text { humur } \\ \text { PISfar } & \text { Safra } & \text { Sufur } \\ \text { PIXDar } & \text { xaDra } & \text { xuDur } \\ \text { PIzrag } & \text { zarga } & \text { zurug }\end{array}$

The symmetry in the plural form is simply a mere reflection of the symmetry in the singular forms.

Derived colour terms behave differently with regard to plural forms. Before delving into this, it is worth mentioning 
that these colours, unless used figuratively, cannot be used to describe humans; they can only be used to describe nonhuman objects. These colour terms have a regular plural form; they constantly use the sound feminine plural which is formed through using the suffix -aat after the feminine form of a noun as shown in (9) below:

(9) The plural forms of derived colours:

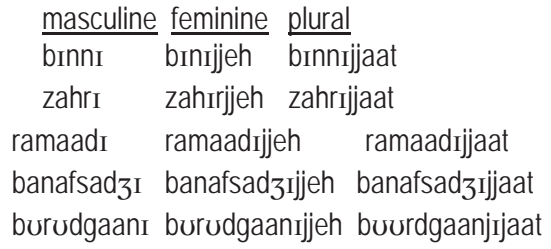

This plural form, which is feminine in gender, can be used to describe both inanimate feminine plural nouns such as saijjaaraat 'cars' and inanimate masculine plural nouns as in ?rbwaab 'doors' The absence of a plural masculine form is accounted for in terms of the fact that non-human plurals can be referred to by a singular feminine form or a plural feminine form. This same way of pluralization is found in standard Arabic. The difference in behaviour can be seen as a reflection of the semantic differences in the sense that only primary colours can be used to describe human beings or attributes related to human beings.

\subsection{Parts of Speech}

Another aspect in which primary colour terms make themselves from derived colour terms is the number of parts of speech each group has. Primary colour terms have a wide range of parts of speech; they have verbs derived from the name of the colour; they have count and noncount nouns; and they have different forms of adjectives with variable meanings. Furthermore, some of these parts of speech have been used metaphorically. This feature of having parts of speech derived from the colour name has been emphasized by Maffi (1990) in her study of Somali Colour terms. She has found that only six colour words were found to have both nominal and verbal forms while the rest of the colours are expressed in the nominal forms.

The parts of speech derived from primary colour terms and their meanings are listed in (9) below:

(9)
a. $21 b j a D$
'white'

verbs: ?I-baj-jiD(vt) (article) 'to make a fair copy of' (face) 'to make one proud because of good deeds' adjective: ?Ib-jaD 'white' ' general description'

?ibjaDaani 'white' ' whitish/physical description of a male'

beiDa 'white/female' general description

(9)

Nouns: bajaaD 'whiteness'

b. Piswad 'black'

Auzaizi:1981)

verbs: ?I-saw-wid(vt) (article)'to write a draft' (face) to cause shame by doing bad deeds' (Al-

adjective: ?Iswad 'black'

sawdaawidi (attitude) pessimistic

?iswaadan ' 'black' blackish/physical description of a male'

nouns: sawaad 'blackness'

miswaddeh 'a draft'

?iswerde 'nervous breakdown' (Al-Auzaizi:1981)

(9)

c. Pithmar 'red'

Verbs hammar (vi) 'became red/caught erysipelas'

hammar (vt) (chicken) 'roast'

Adjectives 2hmar 'red' 
(9)

mham-mir 'sick/ the one who caught erysipelas'

Nouns humrah 'redness/minimal'

houmarah 'lipstick/blushing powder'

abu hmaar 'erysipelas'

hamaar 'redness/ maximal'

d. PiSfar 'yellow'

Verbs Safran (vi) (face) 'his face became yellow/became pale'

ISfarr (vi) (wheat) 'it has become yellow' (Al-Auzaizi:1981)

Adjectives ?iSfar "yellow"

mSafrin (face)'sick/ pale'

Nouns Sufrah 'yellowness/minimal'

'the time before sunset' (Al-Auzaizi:1981)

Safaar 'yellowness/maximal'

?ISfaar 'jaundice' (Al-Auzaizi: 1981)

(9)

e. PIXDar 'green'

Verbs xaDran (vi) 'became rotten'

xaDDar (vt) 'to make green'

Adjectives mxaDrin 'rotten'

mixDarr 'greenly/greenish'

PIXDar 'green'

Nouns xaDaar 'greenness/maximal'

xuDrah 'greenness/minimal'

xuDaar 'vegetables'

xuDrah 'vegetables'

?IXDerrreh 'a kind of plant'

(9)

f. ?izrag 'blue'

Verbs zourag (vi) (face) 'became blue/sick' (Al-Auzaizi: 1981)

Adjectives ?rzrag "blue"

mzourig (face) 'bluish i.e. he is sick'

Nouns ?izrag 'blue'

zaraag 'blueness/maximal'

zurgah 'blueness/minimal'

mizraag 'death i.e. referring to the colour of the dead'

Derived as well as secondary colour terms do not show this versatility in derivations. In fact, most of them appear without any derivation. However, there are some derivatives related to the colour term gray 'ramaadr'. There is a verb, an adjective, and a noun which all can be used to describe a disease and the condition of the person who has this disease. However, one cannot tell whether these derivatives are related to the colour or to the source object i.e 'ash' the colour is related to. The parts of speech are given in (10):

(10) Verbs rammad (vi) 'to have sore eyes'

Adjective ramadi 'gray' mramld 'having sore eyes'

Nouns ramad 'ophthalmia'

This variation in the number of parts of speech can be accounted for in terms of the fact that derivation takes place from a single morpheme in the case of primary colour terms while it is blocked in case of derived colour terms since they are made of two parts. As mentioned before, derived colour terms are made of two parts: A collective noun and a relational suffix. Interestingly enough, except for ramaadi, no parts of speech were derived from derived or the secondary colour terms or the source objects of derived colour terms; there are no verbs, adjectives, or nouns derived from either 
the derived colour terms or their source objects. This phenomenon can be accounted for through two reasons. First: derived colour terms are already derived; a fact which blocks any further derivation. In Arabic one cannot derive a verb from an adjective that is already derived through a suffix. The new verb, had there been one, is supposed to show the source object and the colour meaning. Second, at the semantic level derivation is forbidden since the new verb, had there been one, is ambiguous whether it is related to the source object or the colour. For example, Jordanian Arabic contains the verb ðahhaba 'to make golden' which can be interpreted as related to the colour 'ðahabı' or to gold 'ðahab' itself. I claim that it is most likely related to the material not to the colour. The same explanation applies to the derivatives related to the colour ramadı 'gray' One cannot tell whether these derivatives are related to the original object from which the colour is derived i.e. ramaad 'ash' or to the colour itself..

\subsection{Reduplication}

Reduplication is a form of affixation in which some part of a base is repeated to the left or to the right of the base. Reduplication departs from the traditional view of affixation in the sense that the added part is partially or wholly determined by the base. The reduplicant can be a whole word, a whole morpheme, a syllable or a sequence of syllables or simply a string of consonants and vowels (Spencer, 2000).

Kager (1999) identifies two types of reduplication. Total reduplication involves copying a complete word or the whole base, whereas partial reduplication involves copying part of the base. Both of these types are witnessed in JA. Consider (11) below:

(11)

a. hilwa hilwa 'Complete reduplication'

beautiful beautiful

'She is so beautiful.'

b. hilwa halawa 'Partial reduplication'

beautiful beauty

'She is so beautiful.'

The example (11) b, gives two important remarks about he nature of partial reduplication. First, the two words must be of different parts of speech. In the example, the first word is an adjective while the second is a noun. Second: the two words are derived from the same root i.e. they share the same core consonants. The root for the two words is /h-I-w/

Basic colour terms in JA vary with regard to their behaviour in reduplication; only the first six colour terms in the hierarchy i.e. the primary colour terms, can appear in both types of reduplication as in (12) below:

(12)

a. complete reduplication
?ibjaD ?ibjaD 'white white' very white
?iswad ?iswad 'black black' very black

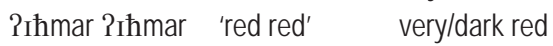
PIXDar ?IXDar 'green green' very/dark green
?ISfar ?iSfar 'yellow yellow' very/dark yellow
?izrag ?izrag 'blue blue' very/dark blue

b. partial reduplication
?rbjaD bajaaD 'white whiteness' very white
?iswad sawaad 'black blackness' very black
?ihmar hamaar 'red redness' very/dark red
PIXDar xaDaar 'green greenness' very/dark green
?iSfar Safaar 'yellow yellowness' very/dark yellow
?izrag zaraag 'blue blueness' very/dark blue

Derived colour terms, on the other hand, do not undergo either type of reduplication. The colour term bunnI, for 
instance, has never been used in reduplication. The case that derived colour terms do not appear in partial reduplication can be partially accounted for in terms of the fact that these colour terms lack an abstract noun which can be used in this structure; they are related to material collective nouns. However, their absence in complete reduplication is not easy to account for. Two scenarios arise in this context. First: if morphologically-complex structures other than colour terms appear in reduplication, while colours do not, then this phenomenon can be colour-bound. Second: if morphologicallycomplex structures do not surface in reduplication, then the absence of reduplicated forms of colour terms can be due to the grammatical process itself. Whether the reason is the first or the second, the data show that there is a division in basic colour terms between primary colour terms and derived ones.

Finally, it is worth mentioning here that in order to emphasize "bunni' as well as all the other derived colour terms, the adjective yaamrg 'dark' is used. Nonetheless, this adjective does not have the positive connotations the reduplication forms have.

\subsection{Frequency}

In order to determine the frequency of colours in our daily life, I decided to quantify the occurrences of colours in proverbs. Proverbs were used for several reasons. First: proverbs are expressions that are likely to occur on a daily basis. Second: proverbs represent the consensus' agreement; they reflect the communities' shared values, traditions, beliefs, and supposedly, their basic colours. Thirdly, proverbs are used unconsciously; they indirectly unfold what the individuals store in their minds.

The corpus of proverbs containing colour terms consists of 83. The proverbs were collected by the Hashemite University students enrolled in a compulsory English course. The frequency of colour terms in the proverbs is given in table (3) below:

Table (3): The frequency of colours in proverbs

\begin{tabular}{|c|c|c|c|}
\hline Colour & & frequency & Percentage \\
\hline PrbjaD & 'white' & 18 & 22.22 \\
\hline ?iswad & 'black' & 17 & 20.98 \\
\hline ?iћmar & 'red' & 15 & 18.51 \\
\hline PIXDar & 'green' & 11 & 13.58 \\
\hline ?iSfar & ‘yellow' & 8 & 9.87 \\
\hline Pizrag & 'blue' & 7 & 8.64 \\
\hline BinnI & 'brown' & 4 & 4.93 \\
\hline zahri & 'pink' & 1 & 1.23 \\
\hline Ramaadı & 'gray' & 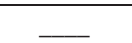 & \\
\hline banafsad3I & 'purple' & 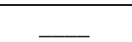 & \\
\hline Burudgaan I & 'orange' & & \\
\hline
\end{tabular}

The table above shows the gradual descent of frequency of colour terms in proverbs. Two major leaps are noteworthy in the table. The first took place between ?IXDar 'green' which is the fourth most frequent colour term (11) and ?iSfar 'yellow' (8). This movement can be accounted for by the fact that the community is an agricultural one in which the colour green is dominant. Moreover, the colour green is remarkably important to the community since it is favorite colour for Prophet Mohammad. I suppose this explanation goes along the lines of Al-Jehani (1990) who has found that colour distinction and realization is deeply affected by the culture and the community the person is living in. The second major leap occurred between ?izrag 'blue' (7) and bInnI 'brown' (4). This leap can be accounted for in terms of the division between primary colour terms and derived ones. In fact, three of the four occurrences of the colour term bınn I 'brown' appeared as ?ismar 'brown' which is restrictively used to describe human complexion. This means that the colour term binn I 'brown' appeared only once. 
The division between the two types of colour terms becomes clear when we realize that the other three colour terms did not occur in proverbs at all. This distinction in frequency goes along the line of the distinction between primary and derived colour terms.

\subsection{Metaphorical Colour Terms in Jordanian Arabic}

Another aspect I sought to examine is the range of metaphorical meanings encoded in each colour term. Colour terms have always been used to refer to emotional states. (Cacciari et.al.:2004 ). This phenomenon seems to be universal. Different cultures seem to use various colours to refer to various emotional states, values, behaviours, traditions, etc. This connection comes as no surprise since the information we acquire through our visual system and that acquired through language are the only two sources of knowledge (Jackendoff: 1992). Furthermore, I claim that resorting to what is culturally stereotyped is one means of facilitating understanding since what is stereotyped is generally shared between the members of the community. (For more on metaphor use and interpretation see Gibbs: 1994 and Zoltan: 2003)

In discussing this issue I decided to utilize proverbs, again. The argument is the following: since these colours are the basic colours in the community and since they are supposedly shared among the members of the community, they must have been used to stand for some values the community appreciates or disguises. In other words, colours are part of the cultural heritage of the community, and ,in this context, they are means of evading saying the obvious or circumventing around the obvious by expressing it through a polite way yet accessible to all the members of the community. I would rather call these meanings figurative meanings of colours since, in most cases, the colour itself does not appear. In his study of the psychological and social connotations of colour terms in Arabic, Omar (1997) provided a list of proverbs in which colours appear from both Standard Arabic (Classical and Modern) and Egyptian Arabic.

I claim that the best data to represent the metaphorical meanings of colours are proverbs due to the fact that they are mostly metaphorical in meaning and due to the fact that they are part of the shared social subconscious of the whole community. A corpus of 81 proverbs has been analyzed. The colours will be handled following the order suggested by Berlin \& Kay (1969).

\subsubsection{White}

Generally speaking, this colour indicates goodness. According to Omar (1997), the colour is used to indicate purity, honesty, optimism, satisfaction, beauty, truce and peace. In JA, the colour term 'white' indicates beauty as in (13). The proverb reflects the Jordanian society's preference for white women.

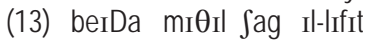

white-she like half the-turnip

'She is so white like the internal side of a piece of turnip.'

Another value encoded in the colour term 'white' is kindheartedness. This usage seems to be universal. The colour term 'white' is used to mean purity and kindness. Consider:

(14) galb-u ?ibjaD

heart-his (be) white

'His heart is white i.e. he is so kind.'

It may also be used to indicate prosperity, richness or days of happiness as in the:

(15) ?Il-ajjaam ?Il biiD

the-days the-white

'The white days i.e. days of prosperity'

In (16) below, the same colour term can be used to refer to silver coins since their colour is white. However, the general meaning of the proverb is to inform the addressee that he was of no benefit to speaker:

(16) maa Jufit minnak ?ibjaD wa la swad

not saw-I from-you white and no black

'You did not give me any silver coins or cents i.e you did not give me any silver or black coins.'

One final meaning for the colour term 'white' is extra money, savings or the money that might be beneficial in future as it might evade a person distress or misery expressed by the colour ?iswad 'black' as in the proverb:

(17) xabbi girfak II-bjaD la youm-ak II-swad

Hide(save) piaster the-white to day-yourthe-black

'Save your white piaster to your black day i.e. save your extra money to days in need.' 


\subsubsection{Black}

One general meaning associated with the colour term black is wickedness, evilness and badness. In this context, Omar (1997) states that the colour term 'black' and its derivatives are considered taboos and consequently avoided. They are either followed by expressions that show the unpleasant meanings of the colour, replaced with other colour terms or modified versions of the colour itself. (For more, see Omar: 1997, 202-205)

The colour term 'black' can, however, be surprisingly used to express beauty especially beauty of eyes since the Arabs prefer eyes which are so white with the iris is so black. This meaning is expressed in the proverb below:

(18) $\mathrm{mI} \int \mathrm{la}$ sawaad Iíuun-u

not for blackness eyes-his

'Not for the blackness of his eyes i.e. it is not for the beauty of his eyes (that I did)

All the other meanings associated with the colour term 'black' are given in (19) below. In (19)a, black is used to indicate 'bad omen' since it is connected to the crow which, in our culture, indicates that something bad will happen. This same idea is reiterated in multiple proverbs. Another meaning is grudge and malice as in (19)b. A third meaning, given in (19)c, is the cloudy weather, or when the sky is overcast with clouds. This meaning can be interpreted at both levels; literal where the colour of the sky looks black or metaphorical where it means a good omen i.e. rain is coming. It can be still used as a bad omen that one needs to take all the precautions to avoid flooding or damage. Finally, the colour term 'black' can also be used to indicate misery or days of need as in (17) above given below as (19)d:

(19)

a. Riswad mi $\theta_{\mathrm{Il}}$ li-yraab

black like the-crow

'(he) is black like the crow i.e. he is a bad omen.'

b. galb-u RIswad mi $\theta$ Il ?Il-guTraan

i. heart-his black like the-tar

ii. 'His heart is like tar i.e. he is malicious and grudging.'

c. ?is-sama dzajjeh min myarrib souda souda

i. the-sky coming from west black black

ii. 'The sky is very cloudy i.e. it is going to rain heavily.'

d. $\quad$ xabbi girfak II-bjaD la youm-ak li-swad

iii. Hide(save) piaster the-white to day-your the-black

'Save your white piaster to your black day i.e. save your extra money to days in need.'

\subsubsection{RED}

Similarly, red can also be used to clarify different targets; different themes can be expressed by using the colour red. Omar (1997) states that the connotations the colour term 'red' expresses can be contradictory. The contradiction takes place because this colour is related to various natural things; some are enjoyable and some are pain-causing. As a result of its relation to blood, the colour is used to express hardness, and danger; as a result of its relation to fire, it is used to express seduction and lust; and as a result of its relation to gold and roses, it is used to express beauty, love and shyness.

In Jordanian Arabic, the colour term 'red' is used to express a variety of themes as well. One meaning for red is grudge and malice as in (20)a, where the red snake is no different from the brown snake except in colour. This proverb can be used to describe two women behaving wickedly and grudgingly. Red can also be used to indicate beauty of women as in (20)b. It can also be used to indicate a great value or dearness as in (20)c and d; finally, it can be used to indicate youth and handsomeness where an old man is not supposed to put on or to wear red clothes as in (20)e:

(20)

a. hajjeh hamra? wo hajjeh samra? snake red and snake brown

'A red snake and a brown snake.'

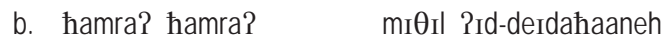


Red red like a wild rose.

"She is like a red rose i.e. she is so beautiful."

c. ?istagbal-na-ah wo farafna-l-u ?issindzeh ?Il-hamra?

received-we-him and furnished-for-him carpet the-red

'We received him and furnished for him the red carpet.'

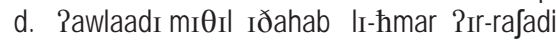

sons-my like the gold the red Rashadi

'My sons are like the red gold (which is the purest).'

f. baid Il-kabrah dzubbah hamra?

after old age robe red

'It is not good for an old man to wear red. Age cannot be covered.'

\subsubsection{Yellow}

This colour term in general has negative connotations. Omar (1997) maintains that the term is connected to weakness, disease and weariness, and that it has never been used to praise. In JA, it may indicate sickness or paleness as in (21)a and b; fakeness as in (21)c and arrogance as in (21)d:

(21)

a. Widzh-ak ISfar mi $\theta$ Il Illermuuneh

face-your yellow like the-lemon

'Your face is like a lemon. i.e. You look sick.'

b. Suu maalak mSafrin

What you yellowish

'What's wrong with you? You look pale! You look sick.'

c. maa kul ISfar diinaar la Sufurt-u

not all yellow dinar for its yellowness

'Not all that is yellow is gold. All that glitters is not gold.'

d. PIiuun-u Sufur

eyes-his yellow

'His eyes are yellow. i.e. He is arrogant and envious.'

\subsubsection{Green}

Green is the only colour that has no contradictory connotations. Omar (1997) maintains that the colour expresses joyful themes which can be regarded as a reflection of the natural close relationship between the colour and joyful objects in life as plants, and jewels. It is usually connected to fertility and blessings.

In JA, the colour term is exceedingly positive. It has three meanings; the first is generosity as in (22):

(22) ma xDar zert- $\mho$

so green oil-his

'His oil is so green i.e. he does not keep his (olive) oil for a long time to become yellow.'

Another meaning for green in JA is success; this meaning is best exemplified in (23) which is usually said by a mother to her son or daughter, or by a wife to her husband, before leaving, praying to Allah that his/her path and accordingly everything s/he does on that day will be a success:

(23) darbak xaDra path-your green

'(I pray to Allah to make) your path green. i.e. to succeed wherever you go."

Green can also be used to mean good omen, fertility and abundance, all combined in one meaning as in:

(24) adaih xathra hands-his green

'His hands are green. 


\subsubsection{Blue}

Generally speaking, the colour blue has negative connotations. In JA, the colour blue has two meanings; the first is literal while the other is figurative. The first meaning involves praising the colour itself especially for eyes. Blue eyes are scarcely seen in Jordan, and they invite the person to scrutinize and to praise them:

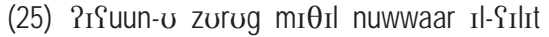

eyes-his blue like blossoms the Silit

'His eyes are so blue like the blossoms of the Silıt (a wild plant); his eyes are so beautiful.'

Obviously, this meaning is not figurative. However, the other meaning of blue is figurative. It is taken for death or lack of health. Blue is the mark of the stoppage of blood running in the veins. For example, the colour of a corpse is nearly blue since there is no life in it, and blood is not running any more to give life. Similarly, when one falls and gets bruised, the colour of the spot gets blue. Then, blue is used to indicate death or near death. However, another reason for using the colour is the universal notions of taboos and euphemism. Death is a universal taboo which all cultures tend to avoid and to replace with other mitigating expressions i.e. euphemisms. Consequently, the word mout which means 'death' in Arabic is replaced by a colour in this context. Although most of the examples here involve threatening, not one of them dares to mention the real word for death frankly:

(26) Dal juxbut fii-h ta xalla dzild-u izrag izrag remain hitting (in)-him until made skin-his blue blue

'He hit him so hard that his skin became so blue.'

Sometimes, the skin may not turn blue at all, but the term is used to indicate that the person was severely spanked. Another example is given in (27) below:

(27) Widzh-e ?izrag zaj Il-mayyet

face- his blue like the-dead

'His face is so blue like a dead person. i.e. either he is sick or not fed well.'

A third meaning expressed by the colour blue is stinginess. Consider the example:

(28) YaDm-uh ?izrag

bones-his blue

'One's bones are blue. i.e. he is so stingy.'

A final note before moving to derived colours is that the scope of this paper is not to detect historically how or when or where these connotations started.

\subsubsection{Derived colours}

Derived colour terms do not exhibit the same variability witnessed in primary colours. In fact, Omar (1997), in his detailed account of the psychological and social connotations encoded in colour terms, did not mention the last five colour terms as containing any connotations. The focus was solely restricted to primary colour terms. The same generalization applies to derived colour terms in Jordanian proverbs. The only colour term that can be excluded from this generalization is the colour brown which appears in two different versions: the first is bIn-nI and the other is ?ismar 'brown'. The latter is restrictively used to literally describe human beings. Thus, the colour term used here has a restricted use. However, linguistically speaking, it behaves in a very similar manner to primary colours. (see Bender: 1983 for other colours used to describe skin in Sudan )

Turning to the connotations of this colour, it can mean grudge and malice as in (20)a. repeated below as (29):

(29) hajjeh hamra? wo hajjeh samra?

Snake red and snake brown

'They are a red snake and a brown snake.'

In the following proverb, it can mean beauty in which case people with brown complexion are seen as responding to white women who are arrogant of their beauty:

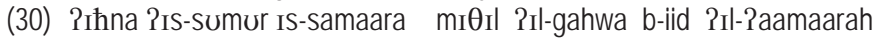

we the-brown(people) like the-coffee in-hands the-princes

'We, the brown people, are like coffee in the princes' hands. i.e. Although we are not white still we are beautiful.'

It can generally mean lack of beauty in which case the brown person must compensate by having a sense of 
humor. A person should not add lack of self-respect to his/her lack of beauty as in:

(31) ?ismar digis wa la ?ibjaD difis

brown humorous and not white rude

'A humorous brown person is better than a rude white one i.e. beauty is in the soul.'

Apparently, the colour brown occurred quite frequently; however, it all occurred in another name which is not classified as the basic colour term. This fact nullifies the inclusion of the colour brown in the primary colours group.

\subsubsection{Variant names}

Most of the time derived colours can be expressed through different names, or sometimes cannot be differentiated from other colour terms. The colour bin-nI 'brown' for example, can be replaced with ?ismar 'brown' and $\hbar I n T_{I}$ ' related to wheat' when it comes to describing people. The colour burudgaanI 'orange' can be mixed with mufmusi 'apricot'. The colour ramaad I 'gray' can be replaced with $2 \pi r S_{a} S_{I}$ 'leadish/related to lead' and satfanI 'the common name for ash'. This phenomenon is not evident in the case of primary colours.

\subsubsection{A Final Remark}

This section is concerned with the colour ?Ismar 'brown'. As mentioned before, this colour is restricted in use to describe human beings as well as wheat bread. This colour is not presented by Berlin and Kay as a basic colour. However, applying the characteristics introduced earlier by the two scholars, we find that this colour abides by two of them. It is morphologically simple; it is salient in everyday language. Nonetheless, its signification is implied by bmnI and it is restricted in use. Moreover, applying the criteria presented in this paper, we find that this term passes them all. First, it can be used in reduplication as in (a) and (b) below. Second: it has a wide range of metaphorical meanings as given above. Third: the feminine form follows the feminine form of the primary colours as in (c) below. Fourth: it can be pluralized in a very similar manner to the primary colour forms as in (d).

(33)
a. 2ismar ?ismar 'very brown'
b. ?ismar samaar 'very brown'
c. ?ISmar 'brown-MAS' samra? 'brown (FEM)'
d. PIsmar (SING.) sumur 'brown (PL.)'

I suggest that this colour term be listed under bInnI as a variant of the basic colour term.

\section{Conclusion}

This paper has set out to apply new criteria to support the dichotomy proposed by Kay \& McDenial (1978) that basic colour terms can be categorized into primary and derived colour terms. The paper has shown that the behaviour of primary colour terms lexically i.e. the parts of speech derived from the colour terms and grammatically i.e. the behaviour of the colour term in some grammatical processes including reduplication, pluralization and derivation is completely different from the behaviour of derived colours.

The paper has found out that, unlike derived colour terms, primary colour terms i.e. white, black, red, green, yellow and blue have verbs, nouns and adjectives derived from them. In addition, they are more likely to be used some grammatical processes such reduplication and pluralization. It has been found that these primary colour terms are more frequent and carry more figurative meanings than derived colour terms do.

The significance of the study is stemmed from its being the first one to provide new criteria for dividing colour terms into two groups, giving some credit to the findings of Berlin and Kay (1969\& 1978). It is also the first in studying the figurative meanings of colour terms in proverbs.

\section{Acknowledgements}

This research is funded by the Deanship of Research in Zarqa University/Jordan.

\section{References}


Al-Adaileh, Belal A. (2012). The connotations of Arabic Colour terms. Linguistics online.

Al-Auzaizi, Ruks Bin Zaid. (1981). The Dictionary of Jordanian Traditions, Dialects, and Peculiarities of speech. Ministry of culture, Jordan.

Al-Jehini, Nasir M. (1990). Colour terms in Mecca: A sociolinguistic perspective. Anthropological Linguistics. 32 (1-2) 163-174

Bender, Lioned, M. (1983). Colour term encoding in a special domain: Sudanese Arabic skin colours. Anthropological Linguistics. 25 (12) $19-27$

Berlin, Brent \& Paul Kay. (1969). Basic Colour Terms: Their Universality and Evolution. Berkeley: University of California Press.

Crawford, T. D. (1982). Defining Basic Colour Terms. Anthropological Linguisitics. 23 (3) 338-343.

Davies, Davies, I. R. L., Roling, P., Corbett, G. G., Xoagub, F. \& Xoagub, J.. (1998).

Colour Terms and Colour Term Acquisition in Damara. The Journal of Linguistic Anthropology, 7(2), 181-207.

Kager, Rene. (1999). Optimality theory. Cambridge: CUP.

Kay, Paul, Brent Berlin and William Merrifield, (1991). Biocultural Implications of Systems of Colour Naming. In Blount, Ben C. (ed.) Language, Culture and Society: A Book of Readings. Illinois: Waveland.

Kay, Paul \& Chad K. McDenial. (1978). The Linguistic Significance of the Meaning of Basic Colour Terms. Language 3:610-46

Maffi, Luisa. 1990. Somali colour term evolution: Grammatical and semantic evidence. Anthropological Linguistics.32 (3-4) 316-334

Moro, Anna L. 2007. An Exploration of colour terms in English-lexifier Atlantic Creoles. Lingua. 117 1448-1461.

Moss, Anthony E. (1989). Basic Colour Terms: Problems and Hypotheses. Lingua (78) 313-320.

Omar, Ahmad Mukhtar. 1997. Language and colour. Palam Ral-Kutub (The world of Books), Egypt.

Rabab'ah, Khalid \& Emad Al-Saidat (2014). Conceptual and Connotative Meanings of Black and White Colours: Examples from Jordanian Arabic. Asian Culture and History 6:2.

Spencer, Andrew. (2000). Morphological theory. Oxford: Blackwell. 